\title{
Outcomes and risk factors for cancer patients undergoing endoscopic intervention of malignant biliary obstruction
}

\author{
Georg-Martin Haag ${ }^{1}$, Thomas Herrmann 1,2,3 , Dirk Jaeger ${ }^{1,5}$, Wolfgang Stremmel ${ }^{2,5}$, Peter Schemmer ${ }^{4,5}$,
} Peter Sauer ${ }^{2}$ and Daniel Nils Gotthardt ${ }^{2,5^{*}}$

\begin{abstract}
Background: Malignant bile duct obstruction is a common problem among cancer patients with hepatic or lymphatic metastases. Endoscopic retrograde cholangiography (ERC) with the placement of a stent is the method of choice to improve biliary flow. Only little data exist concerning the outcome of patients with malignant biliary obstruction in relationship to microbial isolates from bile.

Methods: Bile samples were taken during the ERC procedure in tumor patients with biliary obstruction. Clinical data including laboratory values, tumor-specific treatment and outcome data were prospectively collected.

Results: 206 ERC interventions in 163 patients were recorded. In 43 \% of the patients, systemic treatment was (re-) initiated after successful biliary drainage. A variety of bacteria and fungi was detected in the bile samples. One-year survival was significantly worse in patients from whom multiresistant pathogens were isolated than in patients, in whom other species were detected. Increased levels of inflammatory markers were associated with a poor one-year survival. The negative impact of these two factors was confirmed in multivariate analysis.

In patients with pancreatic cancer, univariate analysis showed a negative impact on one-year survival in case of detection of Candida species in the bile. Multivariate analysis confirmed the negative prognostic impact of Candida in the bile in pancreatic cancer patients.
\end{abstract}

Conclusion: Outcome in tumor patients with malignant bile obstruction is associated with the type of microbial biliary colonization. The proof of multiresistant pathogens or Candida, as well as the level of inflammation markers, have an impact on the prognosis of the underlying tumor disease.

Keywords: Cancer, Pancreatic cancer, Biliary obstruction, Cholangitis, Chemotherapy, Systemic therapy

\section{Background}

Malignant biliary obstruction is a frequent complication in patients with advanced tumor diseases. Both central hepatic metastases and hilar lymph node metastases of gastrointestinal and non-gastrointestinal cancers, but also tumors of the pancreatic head often result in a biliary compression.

\footnotetext{
* Correspondence: Daniel.Gotthardt@med.uni-heidelberg.de

${ }^{2}$ Department of Gastroenterology, Toxicology and Infectious Diseases,

University Hospital Heidelberg, Im Neuenheimer Feld 410, Heidelberg 69120, Germany

5 Liver Cancer Center Heidelberg (LCCH), University Hospital Heidelberg, Im Neuenheimer Feld 460, Heidelberg 69120, Germany

Full list of author information is available at the end of the article
}

Obstructive jaundice is associated with increased morbidity and mortality. Furthermore, intact bilirubin excretion is mandatory for the administration of many chemotherapeutical and targeted agents.

Only limited data exist concerning the role of jaundice in patients with metastatic disease. In a retrospective study published in 1994, median survival in patients with jaundice due to metastatic colorectal cancer was approximately 3-5 months [1]. However, taking into consideration the increasing number of therapeutic agents available for the treatment of metastatic colorectal cancer, more therapeutic options are available after improvement of biliary drainage,

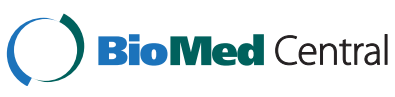

(c) 2015 Haag et al. Open Access This article is distributed under the terms of the Creative Commons Attribution 4.0 International License (http://creativecommons.org/licenses/by/4.0/), which permits unrestricted use, distribution, and reproduction in any medium, provided you give appropriate credit to the original author(s) and the source, provide a link to the Creative Commons license, and indicate if changes were made. The Creative Commons Public Domain Dedication waiver (http://creativecommons.org/publicdomain/zero/1.0/) applies to the data made available in this article, unless otherwise stated. 
resulting in prolonged survival in patients. Furthermore, in tumors being considered as chemorefractory such as hepatocellular carcinoma ( $\mathrm{HCC}$ ), normalization of cholestasis parameters is mandatory to initiate systemic targeted treatment, such as sorafenib [2].

In patients with pancreatic carcinoma, biliary obstruction occurs in approximately 70-90\% of all cases, possibly leading to a deterioration of the patients' general condition due to cholangitis, malabsorption or liver failure [3]. Although surgical bypass has demonstrated a low rate of recurrent jaundice, the surgical procedure itself carries a significant risk of perioperative morbidity and mortality [4].

Endoscopic retrograde cholangiopancreatography (ERCP) has been the preferred method for patients with malignant biliary obstruction since the late 1980s. Studies showed an equivalent success rate and decreased morbidity and mortality for this method in comparison with percutaneous or surgical approaches [5-7]. Yet, in patients with resectable pancreatic cancer, immediate resection of the tumor without a previous ERC is the preferred option [8].

Placement of a plastic or metal stent has been shown to be an effective method in improving biliary flow. Common complications include cholangitis and stent occlusion, whereas pancreatitis is only rarely observed post-ERCP [3]. The optimal type of stent (plastic or metal) is still a matter of debate. Plastic stents have been used as a standard method for biliary drainage; however, regular stent exchange is necessary every $3-4$ months in order to prevent stent obstruction due to biliary sludge. Thus, metal stents are preferred in patients with an expected survival of more than 3 to 4 months [9].

Development of a biofilm is a critical event in the pathogenesis of cholangitis and the blockade of stents. Effectiveness of antibiotic treatment is limited against bacterial colonization of the biofilm [10].

The impact on biliary bacterial or mycotic infection in tumor patients with malignant biliary obstruction on survival is poorly studied. Data from patients undergoing liver transplantation show a negative prognostic impact on the proof of enteric bacteria or Candida species regarding time till organ failure [11-13].

In this prospective, observational study we aimed to identify the spectrum of bactobilia and fungobilia in patients with malignant obstruction, and to explore the association of this spectrum with clinical outcome.

\section{Patients and methods}

The prospective, observational study was conducted at the Center for Endoscopy of the University Hospital Heidelberg in collaboration with the Department of Gastroenterology and the Department of Medical Oncology at the National Center for Tumor Diseases, Heidelberg. Patients were recruited consecutively. Data of patients undergoing ERC for malignant biliary obstruction were recorded in a prospective database. Biliary drainage was performed whenever technically possible.

Bile samples were obtained after selective intubation before any therapeutic procedure was performed. When bile could not be aspirated directly after cannulation, a small amount of sterile saline $(2-4 \mathrm{ml})$ was applied and aspiration was reattempted. Aliquots of all biliary samples were placed in a sterile glass tube containing medium for anaerobic and aerobic bacterial cultures. The material was delivered to the microbiology laboratory within $2 \mathrm{~h}$ of collection and cultured aerobically and anaerobically according to standard laboratory protocols.

The underlying tumor disease including the stage of the disease, the proof of bacteria or fungi, as well as laboratory values including bilirubin and C-reactive protein (CRP) at the time of intervention were recorded. The previous or subsequent application of systemic therapy (e.g. chemotherapy) was documented.

Assuming that the biliary infection would rather influence the short-term outcome whereas the long-term outcome is determined by the underlying tumor disease, patients' outcome was followed for a maximum of one year, patients lost to follow-up were censored at the time of the last documented contact. All patients provided informed consent, the study was performed in accordance with the Declaration of Helsinki. The study was approved by the ethical committee of the University of Heidelberg.

Table 1 Patient characteristics

\begin{tabular}{ll}
\hline Patient characteristics: & 163 \\
Number of patients: & 206 \\
Number of interventions: & $66(36-96)$ \\
Median age (range) & Number of patients (\%) \\
Tumor diagnosis & $60(37)$ \\
Pancreatic carcinoma & $34(21)$ \\
Cholangiocellular carcinoma & $19(12)$ \\
Colorectal carcinoma & $9(6)$ \\
Hepatocellular carcinoma & $8(5)$ \\
Gastric cancer & $8(5)$ \\
Breast cancer & $6(4)$ \\
Neuroendocrine tumor of the digestive tract & $5(3)$ \\
Tumor of unknown origin & $3(2)$ \\
Ovarian carcinoma & $2(1)$ \\
Intraductal papillary mucinous neoplasm (IPMN) & $1(1)$ \\
Lymphoma & $8(5)$ \\
Other tumors & \\
Laboratory parameters before ERC & Median (SD) \\
Bilirubin (mg/dl) & $4.5(6.83)$ \\
CRP (mg/l) & $36.2(62.11)$ \\
\hline
\end{tabular}


Table 2 Microbial isolation

\begin{tabular}{ll}
\hline Organism & Number of patients (\%) \\
\hline Bacteria & $36(18)$ \\
Streptococci, alpha- and beta-hemolytic & $35(17)$ \\
Enterococcus spp. & $27(13)$ \\
Escherichia coli & $27(13)$ \\
Coagulase-negative Staphylococcus & $14(7)$ \\
Klebsiella spp. & $1(1)$ \\
Clostridium & $45(22)$ \\
Other bacteria & $50(24)$ \\
Sterile bile & $28(14)$ \\
Missing data & \\
Multiresistant bacteria & $4(2)$ \\
Vancomycin-resistant Enterococcus faecium & $4(2)$ \\
E. coli with extended spectrum beta-lactamase & $14(7)$ \\
Any multiresistant pathogen & \\
Fungi & $32(16)$ \\
Candida albicans & $11(5)$ \\
Candida glabrata & $76(37)$ \\
Missing data &
\end{tabular}

ERCP was performed in an inpatient setting. Concurrent antibiotic treatment or peri-interventional prophylaxis were performed at the discretion of the responsible physician.

\section{Statistical analysis}

Continuous data were compared using the nonparametric Mann-Whitney $U$ test. Frequency differences were compared using the chi-squared test or Fisher's exact test where appropriate. The actuarial survival rate was estimated using the Kaplan-Meier product limit estimator. Differences between the actuarial estimates were tested using the log rank test. Cox regression analysis was performed for multivariate analysis. Differences were considered significant if $\mathrm{p}$ was $<0.05$. All analyses were performed using PASW Statistics 21.0 (SPSS Inc., Chicago, IL).

\section{Results}

\section{Patient characteristics}

Between October 2006 and December 2008, a total of 163 patients with advanced cancer diseases undergoing ERC were recorded.

The most frequent tumors were advanced pancreatic carcinoma (37\%), followed by cholangiocarcinoma (21\%) and metastatic colorectal carcinoma (12\%). The median age was 66 years (range 36-96 years). Patient characteristics are shown in Table 1.

Among patients with pancreatic cancer, locally advanced disease was documented in $58 \%$ of the cases, metastatic disease in $42 \%$.

A total of 206 ERCP interventions were performed due to suspected biliary obstruction. Biliary drainage with a stent was performed in 143 interventions (69.4\%).

\section{Microbiological results}

Bacterial colonization of the bile was common; overall, bacteria were detected in 128 samples (62\%). The most commonly isolated species were Streptococcus species (36 samples, $18 \%$ ), followed by Enterococcus species

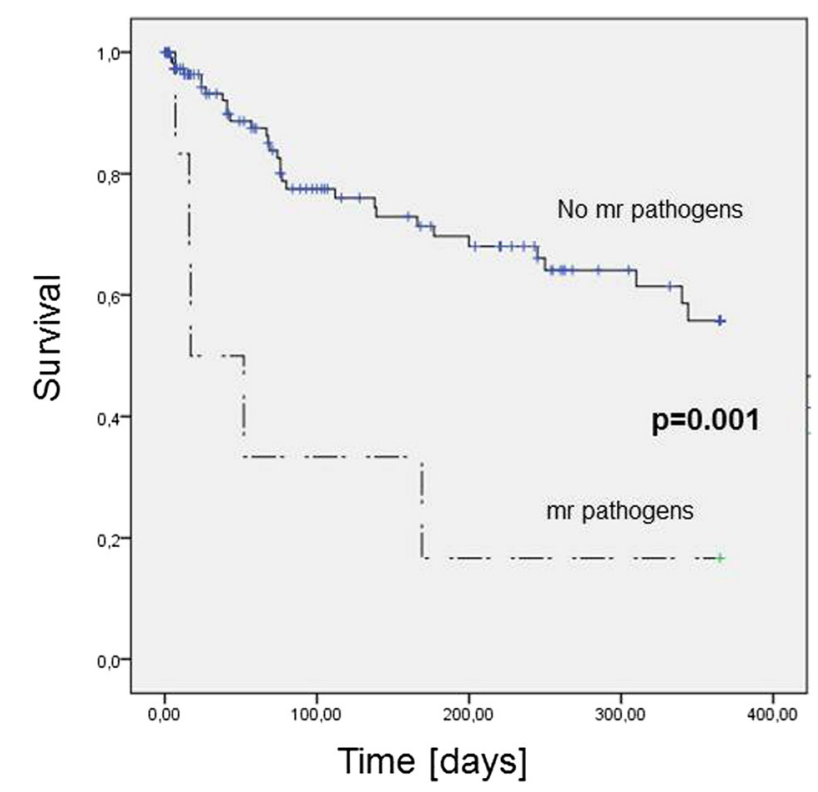

One year survival rate: $55.8 \%$ vs. $16.7 \%$

Fig. 1 One-year survival in tumor patients according to the microbial isolation of multiresistent (mr) pathogens 


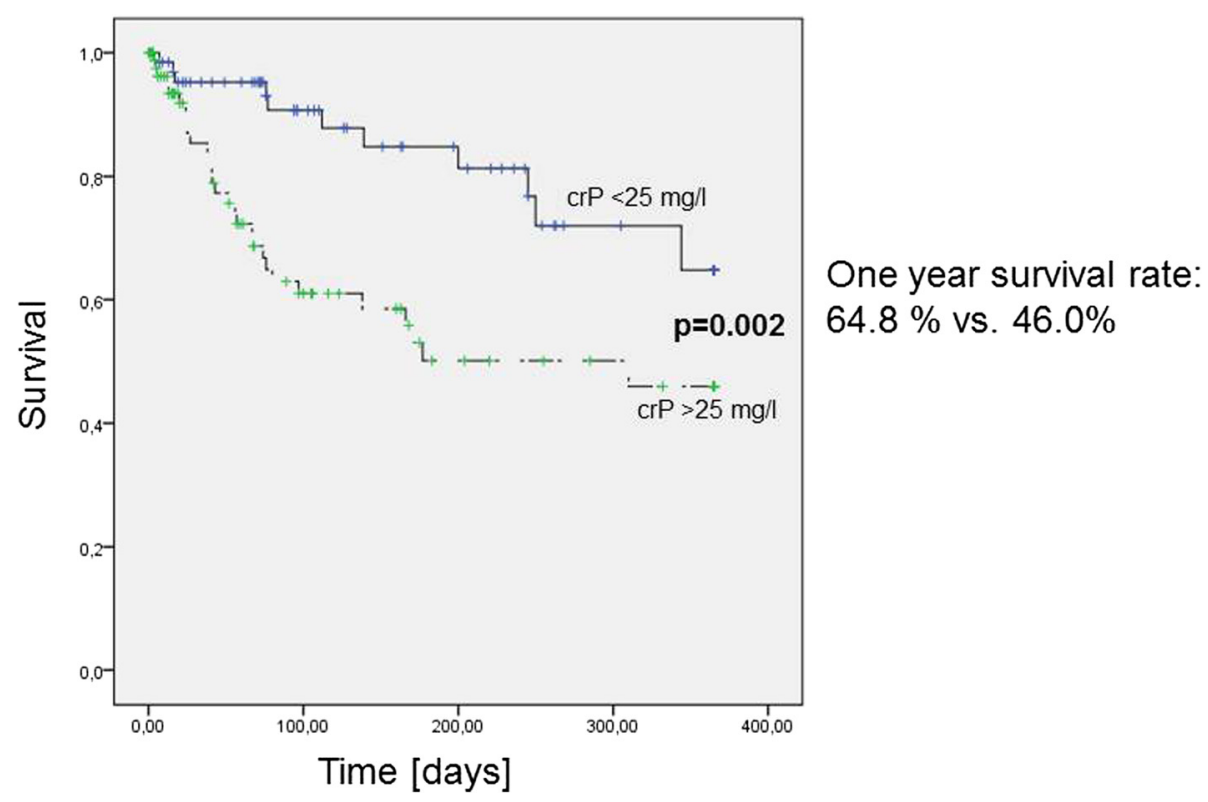

Fig. 2 One-year survival in tumor patients according to preinterventional C-reactive protein level

(35 samples, $17 \%$ ) and Escherichia coli and coagulasenegative Staphylococcus species (27 samples, $13 \%$ respectively) (Table 2).

Multiresistant gram-negative or gram-positive microorganisms, such as methicillin-resistant Staphylococcus aureus or vancomycin-resistant Enterococcus, were only rarely observed; these were detected in 14 samples (7\%).

Mycologic results were available for 130 interventions. Candida albicans was detected in 32 samples (16\%) and Candida glabrata was detected in 11 samples (5\%). Among patients with previous ERC, a significantly increased risk of a fungal biliary colonization was observed (10 of 73 patients without previous ERC vs. 33 of 62 patients with previous ERC, $p<0.001)$.

Patients with proven fungobilia had a significantly longer period of antibiotic therapy before ERC intervention (median 6 days vs. 3 days; $p=0.020$ ).

Table 3 Multivariate analysis of all tumor patients

\begin{tabular}{|c|c|c|c|}
\hline & p-value & $\begin{array}{l}\text { Hazard } \\
\text { Ratio }\end{array}$ & $\begin{array}{l}95 \% \text { Confidence } \\
\text { Intervall }\end{array}$ \\
\hline Placement of Stent (yes vs. no ${ }^{a}$ ) & 0.245 & 2.12 & {$[0.60 ; 7.55]$} \\
\hline Pancreatic primary tumor (yes vs. no ${ }^{a}$ ) & 0.714 & 1.22 & {$[0.43 ; 3.50]$} \\
\hline Bilirubin ( $\geq 7$ mg/dl vs. $<7$ mg/dla ) & 0.321 & 0.58 & {$[0.20 ; 1.69]$} \\
\hline Age ( $\geq 65$ years vs. $<65$ years ${ }^{\mathrm{a}}$ ) & 0.245 & 0.56 & {$[0.21 ; 1.49]$} \\
\hline Proof of candida (yes vs. no ${ }^{\mathrm{a}}$ ) & 0.709 & 1.19 & {$[0.47 ; 3.04]$} \\
\hline $\begin{array}{l}\text { Proof of multiresistant pathogen } \\
\text { (yes vs. no }{ }^{\mathrm{a}} \text { ) }\end{array}$ & 0.009 & 8.77 & {$[1.70 ; 45.17]$} \\
\hline $\operatorname{CRP}\left(\geq 25 \mathrm{mg} / \mathrm{l}\right.$ vs. $\left.<25 \mathrm{mg} / \mathrm{l}^{\mathrm{a}}\right)$ & 0.041 & 2.98 & {$[1.05 ; 8.50]$} \\
\hline
\end{tabular}

areference

\section{Clinical outcome}

In our series, patient outcome was associated with the microbial pathogens involved and the inflammatory state prior to ERC intervention.

Patients with a multiresistant pathogen isolated in bile during the first ERC intervention had a significantly worse one-year survival than patients without finding of a multiresistant species (One year survival rate $55.8 \%$ vs. $16.7 \%$, $p=0.001$ ) (Fig. 1).

Malignant obstruction, the resulting increased risk of cholangitis, and the advanced tumor diseases themselves can all contribute to a permanent state of inflammation. Distinguishing patients with a tumor-related inflammatory state from those with bacterial infection can be a clinical challenge. In our series, tumor patients presenting with increased levels of inflammatory markers at the first ERC intervention, defined by a C-reactive protein level higher than $25 \mathrm{mg} / \mathrm{l}$ (normal range, 0.5-5 mg/l), had a significantly worse one-year survival than patients without increased inflammation markers (One year survival rate $64.8 \%$ vs. $46.0 \%, p=0.002$ ) (Fig. 2).

Multivariate analysis including age (less or more than 65 years), the proof of any multiresistant pathogen, the proof of Candida species, placement of a stent, the primary tumor (pancreatic vs. non-pancreatic), a high bilirubin level (higher than $7 \mathrm{mg} / \mathrm{dl}$ ) and a high CRP level (higher than $25 \mathrm{mg} / \mathrm{l}$ ) confirmed the negative impact of a multiresistent pathogen (Hazard Ratio 8.77, $p=0.009$ ) or a high CRP level (Hazard Ratio 2.98, $p=0.041$ ) (Table 3).

Among patients with pancreatic cancer, those in whom Candida species was found at the first ERC intervention 


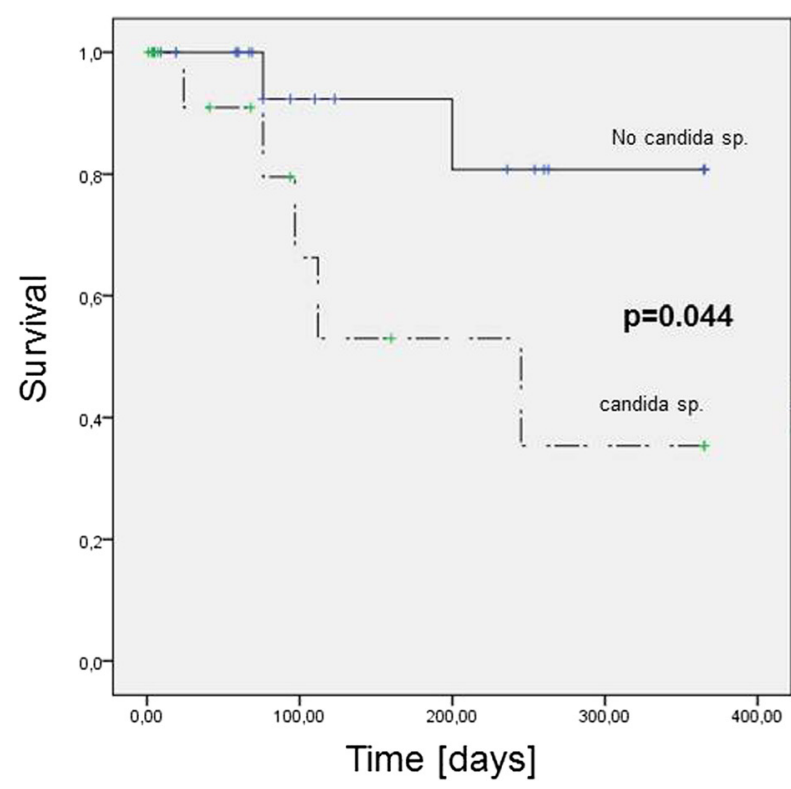

One year survival rate:

$80.8 \%$ vs. $35.4 \%$

Fig. 3 One-year survival in patients with pancreatic cancer according to the proof of Candida sp.

had a significantly lower one-year survival than patients without findings of Candida in bile (One year survival rate $80.8 \%$ vs. $35.4 \%, p=0.044$ ) in univariate analysis (Fig. 3). Additionally, the pre-interventional bilirubin and C-reactive protein level at the first intervention did not differ significantly between these 2 groups.

Multivariate analysis including the proof of Candida species, placement of a stent, tumor stage (locally advanced vs. metastatic disease), a high bilirubin level (higher than $7 \mathrm{mg} / \mathrm{dl}$ ) and a high CRP level (higher than $25 \mathrm{mg} / \mathrm{l}$ ) confirmed the negative prognostic impact of the proof of Candida in the bile. (Hazard Ratio 23.07, $p=0.049$ ) (Table 4).

\section{Previous and further tumor-specific treatment}

59 patients $(36 \%)$ presenting with advanced disease had received previous systemic therapy for their tumor disease, which had to be discontinued because of progressive jaundice. After the intervention, tumor-specific systemic treatment was (re)-initiated in 70 patients (43\%), after the malignant obstruction was alleviated due to successful drainage (Table 5).

The prevalence of candida did not differ between pancreatic cancer patients with or without previous systemic chemotherapy $(p=0.661)$; in addition there was no statistical difference with regard to fungobilia in the rate of patients undergoing systemic treatment after successful ERC intervention ( $p=0.631)$ or in the time till (re-)initiation of systemic chemotherapy $(p=0.161)$.

\section{Discussion}

Data concerning the optimal management and outcome of patients with malignant bile duct obstruction are limited. For more than 20 years, ERC has been the method of choice to improve biliary drainage. Early intervention is desirable to prevent secondary complications such as liver failure or cholangitis. Additionally, an increase in the performance score can often be observed after relief of jaundice.

In our series, the detected bacterial species were common pathogens, which could be controlled with standard antibiotic treatment. In contrast to other cohorts (e.g. patients who underwent liver transplantation [11-13]), multiresistant bacteria were only rarely observed, but these patients had a significantly worse outcome.

Among all cancer patients, those with increased Creactive protein levels had a significantly worse outcome than those with low levels of inflammatory markers. In concordance to our data Iwasaki et al. showed that an inflammation-based prognostic score (mGPS, modified Glasgow Prognostic Score) including C-reactive protein and hypalbuminemia is a significant predictor of postoperative survival in patients undergoing palliative surgery for malignant biliary obstruction [14]. It is unknown

Table 4 Multivariate analysis of pancreatic tumor patients

\begin{tabular}{lccl}
\hline & p-value & $\begin{array}{l}\text { Hazard } \\
\text { Ratio }\end{array}$ & $\begin{array}{l}\text { 95 \% Confidence } \\
\text { Intervall }\end{array}$ \\
\hline Placement of Stent (yes vs. no ${ }^{\mathrm{a}}$ ) & 0.830 & 0.80 & {$[0.11 ; 5.90]$} \\
Bilirubin $\left(\geq 7 \mathrm{mg} / \mathrm{dl}\right.$ vs. $<7 \mathrm{mg} / \mathrm{dl}^{\mathrm{a}}$ ) & 0.982 & 0.98 & {$[0.14 ; 6.78]$} \\
Proof of candida (yes vs. no $\left.{ }^{\mathrm{a}}\right)$ & 0.049 & 23.07 & {$[1.01 ; 526.64]$} \\
CRP $\left(\geq 25 \mathrm{mg} / \mathrm{l}\right.$ vs. $<25 \mathrm{mg} / \mathrm{l}^{\mathrm{a}}$ ) & 0.293 & 0.25 & {$[0.02 ; 3.31]$} \\
metastatic vs. locally adv. disease & 0.108 & 8.92 & {$[0.62 ; 128.38]$} \\
\hline
\end{tabular}

areference 
Table 5 Previous and further treatment

\begin{tabular}{ll}
\hline Systemic therapy before the first ERC & Number of patients (\%) \\
Yes & $59(36)$ \\
No & $92(56)$ \\
Unknown & $12(7)$ \\
Systemic therapy after ERC intervention & \\
Yes & $70(43)$ \\
No & $36(22)$ \\
Unknown & $57(35)$ \\
\hline
\end{tabular}

whether these increased markers are a sign of a more severe bacterial cholangitis or the result of a tumor-related inflammatory status due to a more advanced disease. Other more specific markers like procalcitonin could be useful in distinguishing between a bacterial infection and a tumor-related inflammatory state [15].

The prognostic role of fungi detected in the bile of patients with malignant biliary obstruction is not yet well defined. In our series, Candida species was detected in approximately $25 \%$ of all pancreatic cancer patients; if Candida species was detected in the bile, the patients had a significantly worse one-year survival than those in whom Candida was not detected. The negative prognostic impact was confirmed in multivariate analysis. The reason for this effect is unknown; proof of Candida was neither associated with previous immunosuppressive therapy (cytotoxic chemotherapy) nor with increased levels of inflammatory markers or cholestasis parameters. These findings were in contrast to those for the rest of the patients who had malignant jaundice. No significant difference was noted in the rate of Candida detection between the groups with locally advanced and metastatic cancer. Candida might therefore have a negative impact on outcome in patients with pancreatic cancer and malignant biliary obstruction. A randomized trial with antimycotic treatment in this cohort might help in defining the pathognomonic role of this species in the bile.

With regard to previous and post-interventional tumorspecific therapy, more than one-third of our patients had received previous tumor-specific medical therapy, which had to be discontinued because of tumor progression with obstructive jaundice. After the ERC intervention, a start or re-initiation of tumor-specific therapy is documented to have occurred in $43 \%$ of patients, highlighting the widening oncological therapeutic options once the biliary drainage is improved.

\section{Conclusions}

In summary, endoscopic intervention for cancer patients is a feasible and effective method of improving biliary drainage. It should be performed as soon as malignant obstruction is diagnosed in order to permit the start or continuation of tumor-specific therapy. The bacterial species found in the bile of cancer patients were mostly common pathogens, which could be controlled with standard antibiotic treatment.

Our data showed a negative impact of Candida on oneyear survival in patients with pancreatic cancer. Whether an antimycotic treatment could overcome this negative outcome is unknown. Thus, the role of Candida in the bile should be explored in a randomized trial.

\section{Abbreviations \\ CRP: C-reactive protein; ERC(P): Endoscopic retrograde cholangio(pancreato) graphy; HCC: Hepatocellular carcinoma; mGPS: modified Glasgow Prognostic} Score.

\section{Competing interests}

The authors declare that they have no competing interests.

\section{Authors' contributions}

GMH, TH, DJ, WS, PSch, PS and DNG were involved in the study concept. GMH, $\mathrm{TH}, \mathrm{PS}$, DNG designed the study. GMH, PS and DNG collected the clinical data. GMH and DNG performed the data quality control. GMH, PS and DNG performed the data analysis. The manuscript was edited by GMH, TH, PS and DNG. All authors read and approved the final manuscript.

\section{Author details}

${ }^{1}$ Department of Medical Oncology, National Center for Tumor Diseases, University Hospital Heidelberg, Im Neuenheimer Feld 460, Heidelberg 69120, Germany. ${ }^{2}$ Department of Gastroenterology, Toxicology and Infectious Diseases, University Hospital Heidelberg, Im Neuenheimer Feld 410, Heidelberg 69120, Germany. ${ }^{3}$ Department of Internal Medicine I, Gastroenterology, Hematology and Medical Oncology, Hospital Heide, Heide 25746, Germany. ${ }^{4}$ Department of Surgery, University Hospital Heidelberg, Im Neuenheimer Feld 110, Heidelberg 69120, Germany. ${ }^{5}$ Liver Cancer Center Heidelberg (LCCH), University Hospital Heidelberg, Im Neuenheimer Feld 460, Heidelberg 69120, Germany.

Received: 13 August 2015 Accepted: 25 November 2015 Published online: 04 December 2015

\section{References}

1. Lo CY, Lai EC. Palliation for extrahepatic biliary obstruction by metastatic colorectal carcinoma. Am J Gastroenterol. 1994;89:1852-5.

2. Lin S, Hoffmann K, Schemmer P. Treatment of hepatocellular carcinoma: a systematic review. Liver Cancer. 2012;1:144-58.

3. Das A, Sivak Jr MV. Endoscopic palliation for inoperable pancreatic cancer. Cancer Control. 2000;7:452-7.

4. Van den Bosch RP, van der Schelling GP, Klinkenbijl JH, Mulder PG, van Blankenstein M, Jeekel J. Guidelines for the application of surgery and endoprotheses in the palliation of obstructive jaundice in advanced cancer of the pancreas. Ann Surg. 1994;219:18-24.

5. Smith AC, Dowsett JF, Russell RC, Hatfield AR, Cotton PB. Randomised trial of endoscopic stenting versus surgical bypass in malignant low bileduct obstruction. Lancet. 1994:344:1655-60

6. Speer AG, Cotton PB, Russell RC, Mason RR, Hatfield AR, Leung JW, et al. Randomised trial of endoscopic versus percutaneous stent insertion in malignant obstructive jaundice. Lancet. 1987;2:57-62

7. Shepherd HA, Royle G, Ross AP, Diba A, Arthur M, Colin-Jones D. Endoscopic biliary endoprosthesis in the palliation of malignant obstruction of the distal common bile duct: a randomized trial. Br J Surg. 1988;75:1166-8

8. Van der Gaag NA, Rauws EA, van Eijck CH, Bruno MJ, van der Harst E, Kubben FJ, et al. Preoperative biliary drainage for cancer of the head of the pancreas. N Engl J Med. 2010;362:129-37.

9. Moss AC, Morris E, Mac MP. Palliative biliary stents for obstructing pancreatic carcinoma. Cochrane Database Syst Rev. 2006:2:CD004200.

10. Sung JY, Leung JW, Shaffer EA, Lam K, Costerton JW. Bacterial biofilm, brown pigment stone and blockage of biliary stents. J Gastroenterol Hepatol. 1993;8:28-34. 
11. Gotthardt DN, Weiss KH, Rupp C, Bode K, Eckerle I, Rudolph G, et al. Bacteriobilia and fungibilia are associated with outcome in patients with endoscopic treatment of biliary complications after liver transplantation. Endoscopy. 2013:45:890-6.

12. Kawecki D, Chmura A, Pacholczyk M, Lagiewska B, Adadynski L, Wasiak D, et al. Bacteria isolated from bile samples of liver recipients in the early period after transplantation: epidemiology and susceptibility of the bacterial strains. Transplant Proc. 2007:39:2807-11.

13. Bert F, Larroque B, Paugam-Burtz C, Janny S, Durand F, Dondero F, et al. Microbial epidemiology and outcome of bloodstream infections in liver transplant recipients: an analysis of 259 episodes. Liver Transpl. 2010;16:393-401.

14. Iwasaki Y, Ishizuka M, Kato M, Kita J, Shimoda M, Kubota K. Usefulness of an inflammation-based prognostic score (mGPS) for predicting survival in patients with unresectable malignant biliary obstruction. World J Surg. 2013:37:2222-8.

15. Schuttrumpf S, Binder L, Hagemann T, Berkovic D, Trumper L, Binder C. Utility of procalcitonin concentration in the evaluation of patients with malignant diseases and elevated C-reactive protein plasma concentrations. Clin Infect Dis. 2006:43:468-73.

\section{Submit your next manuscript to BioMed Central} and we will help you at every step:

- We accept pre-submission inquiries

- Our selector tool helps you to find the most relevant journal

- We provide round the clock customer support

- Convenient online submission

- Thorough peer review

- Inclusion in PubMed and all major indexing services

- Maximum visibility for your research 